\title{
Third integer resonance extraction with presence of higher multipoles
}

\author{
V. Nagaslaev* \\ Fermilab, P.O. Box 500, Batavia, Illinois 60510, USA \\ K. A. Brown \\ C-AD Department, BNL, Upton, New York 11973-5000, USA \\ M. Tomizawa \\ KEK, High Energy Accelerator Research Organization, Oho 1-1, Tsukuba-Shi, Ibaraki 305-0801, Japan
}

(Received 26 October 2018; published 4 April 2019)

\begin{abstract}
The slow resonant extraction of a beam in a storage ring is a very well-established technique. Several high-energy physics accelerator facilities around the world are currently using this technique for highintensity beam operations. As the demand for beam power is constantly growing, extraction efficiency in many cases is becoming a limiting factor. In this report, we discuss a new effective approach to improving the slow extraction efficiency by using configurations of higher multipoles. We will describe this approach, determine optimal multipole configurations, and present the results of simulations to support the proposed recipe. We found that, with the use of higher multipole field content, the extraction efficiency can be improved, and, therefore, the level of prompt and residual radioactivity in the accelerator components and surrounding buildings can be reduced by as much as $40 \%$ or more. We also explored manipulating the higher-order effects produced in the pure sextupole configurations for the same purpose and demonstrated that similar results can be achieved by only rearranging the sextupole magnets in the lattice.
\end{abstract}

DOI: 10.1103/PhysRevAccelBeams.22.043501

\section{INTRODUCTION}

For many decades, the complex multipole fields in storage rings have been studied in the context of slow extraction. The literature on this subject is rich and covers half a century of journal reports and conference proceedings, as well as a great number of internal reports. A nice overview can be found, for example, in Ref. [1]. Here, we would like to focus on the concept of increasing the step size while keeping the beam size confined in the extraction acceptance channel by using higher-order multipoles. This discussion is not new and appears first in the literature, as far as we could determine, in a 1997 conference report [2]. Attention to this technique has grown again recently, as can be seen in Refs. [3-5]. It also has been actively discussed at the most recent International Workshop on Slow Extraction at CERN [6]. We expect to see a nice overview of the most recent CERN studies in the upcoming thesis [7]. The detailed analytical treatment of complex multipole fields is nontrivial. In earlier publications, a simplified

\footnotetext{
*vnagasl@fnal.gov
}

Published by the American Physical Society under the terms of the Creative Commons Attribution 4.0 International license. Further distribution of this work must maintain attribution to the author(s) and the published article's title, journal citation, and DOI. assumption was implicitly made that all multipoles can be grouped at one location (i.e., virtual multipole technique). We are using a different approach, which, on the other hand, simplifies finding an optimal placement for additional multipoles in the ring. The study is aimed at improving the multipole configurations at the Japan Proton Accelerator Research Complex (J-PARC) Main Ring (MR) and the Fermilab Muon Campus Delivery Ring (DR). The J-PARC accelerator complex has reached an outstanding extraction efficiency at $99.5 \%$ [8], and they need to go further as they look to raise the beam power up to $100 \mathrm{~kW}$ [9]. The new Mu2e facility at Fermilab [10] is planning to run with a beam power of $8 \mathrm{~kW}$, but their beam loss requirements are very strict due to historically shallow shielding of the former antiproton source buildings.

The Fermilab DR lattice offers a regular structure and a simpler case for nonlinear dynamic studies. Here, we present the results of case studies for the DR as a proof of principle. Similar studies with the J-PARC MR lattice will follow in later publications.

\section{DELIVERY RING LATTICE}

The Fermilab Delivery Ring geometry has a threefold symmetry, as can be seen in Fig. 1. There are three straight sections and three arcs. The full ring length is approximately $505 \mathrm{~m}$. The 114 quadrupole magnets are equally 


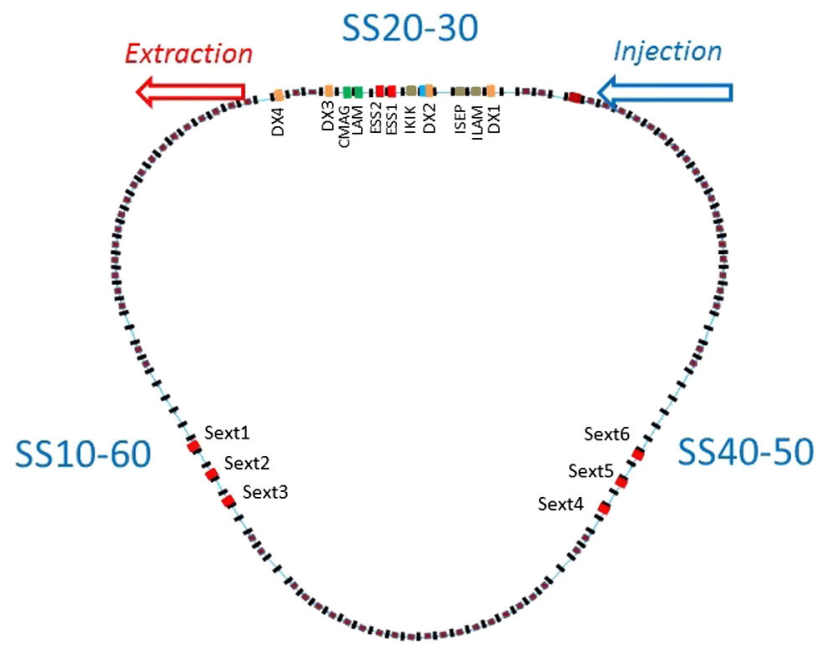

FIG. 1. Delivery Ring and location of the sextupole magnets.

spaced around the ring, forming a regular FODO structure with a nearly $60^{\circ}$ phase advance per cell. Each second-tolast cell in the arcs has missing bend magnets, thus canceling the dispersion in the straight sections. The main optics functions in one section between the arc centers (one period) are shown in Fig. 2. The detailed description of the DR lattice can be found elsewhere [10,11]. Fine-tuning of local functions has been performed in the past to optimize the machine acceptance and phase advances between the stochastic cooling elements for the antiproton collection. In the tracking simulations described here, we used the modified model with the new horizontal tune of 9.65 , although some beta wave, seen in Fig. 2, remains.

Injection (magnetic septa ISEP and ILAM and kicker IKIK) and extraction (electrostatic septa ESS1 and ESS2, magnetic septa LAM and CMAG, and orbit bump dipoles
DX1-DX4) devices are all located in straight section SS20-30, making this section very crowded. Tune ramping quadrupoles are placed in the middle of each of three straight sections (not shown in the picture). Two sextupole circuits with three sextupoles in each are placed in straight sections SS10-60 and SS40-50. The design considerations for this configuration choice can be found in Ref. [12]. Sextupoles are placed near the focusing quadrupoles, which maximizes their driving contributions. Because of the $60^{\circ}$ phase advance, those contributions add up with the proper choice of polarity. Driving contributions of the two circuits are almost orthogonal to each other, which is convenient for tuning the resonance term phase. As will be shown later, this configuration is very effective in suppressing the higher-order effects.

\section{TRACKING SIMULATIONS}

A simple 2D tracking simulation code has been established to study the phase space formation in slow extraction. Multipole magnets (six sextupoles plus any additional multipoles added for this study) were represented with thin lenses with the appropriate kick to the particle trajectories:

$$
\Delta x=0, \quad \Delta x^{\prime}=\frac{1}{m !} \frac{B^{(m)} L}{B \rho} x^{m},
$$

where $m$ is the multipole order ( $m=2$ for sextupoles, $m=3$ for octupoles, etc.), $B^{(m)}$ is the $m$ th derivative of the vertical $B$-field component in $x, B \rho$ is the beam rigidity, and $L$ is the magnet length. The transport between the multipoles and the reference point was performed as a simple matrix transformation. This provides a clean way to see the effects of a selected configuration, separated from other

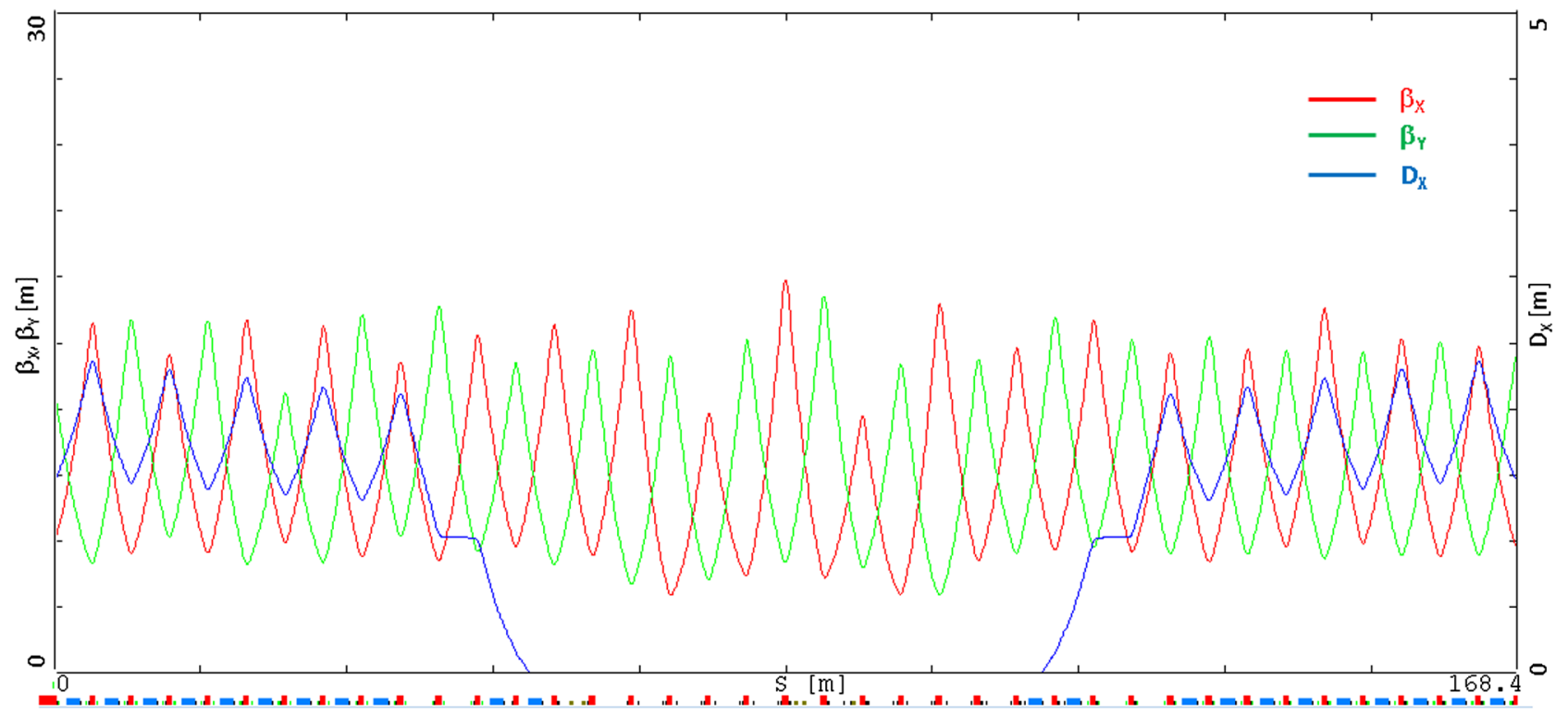

FIG. 2. Lattice functions in one-third of the Delivery Ring. 
nonlinear elements in the machine. Coupling and chromatic effects are neglected in this tracking.

A sample of $N_{p}$ particles was generated using a Gaussian distribution with the rms in $x$ and $x^{\prime}$ corresponding to the Delivery Ring emittance [10]. First, this sample is tracked over 30 turns around the ring to remove the long tails of the beam distribution. Then, we analyze the phase space distribution during the following $N_{t}$ turns. During this time, the tune is fixed, typically at 9.65 , so this is giving us a snapshot of extraction at the beginning of the spill.

\section{CONSTRAINTS OF THE SEXTUPOLE EXTRACTION}

Unlike in the second-order resonant extraction, there are no higher-order fields needed in the conventional thirdorder slow extraction, and, once those fields are present, they are often considered an unwanted contamination. Let us consider the classical Hamiltonian in the presence of the sextupole field in 2D phase space:

$$
H\left(x, x^{\prime}, s\right)=\frac{1}{2}\left(x^{\prime 2}+K_{x} x^{2}\right)+\frac{S(s)}{6} x^{3} .
$$

In the first order of the perturbation theory (PT), this is commonly expanded into the set of azimuthal harmonics and transformed to the other set of canonical coordinates of action $J$ and Floquet phase $\phi$ :

$$
\begin{aligned}
H_{1}(\phi, J, \theta)= & \nu \cdot J+(2 J)^{1.5} \sum_{n}\left[G_{3, n} \cos \left(3 \phi-n \theta+\xi_{3, n}\right)\right. \\
& \left.+3 G_{1, n} \cos \left(\phi-n \theta+\xi_{1, n}\right)\right],
\end{aligned}
$$

where $\nu$ is the horizontal machine tune, $G_{k, n}$ and $\xi_{k, n}$ are the modules and phases, respectively, of the complex azimuthal harmonics $\hat{G}_{k, n}$ of the sextupole field in the storage ring:

$$
\hat{G}_{k, n}=G_{k, n} \cdot e^{i \xi_{k, n}}=\frac{1}{48 \pi} \oint S(s) \beta(s)^{1.5} e^{i \cdot[k \cdot \mathcal{\chi}(s)-(k \nu-n) \theta(s)]} d s
$$

with $k=1$ or 3 , and $\chi(s)=\int \frac{d s}{\beta(s)}, \theta=\frac{2 \pi \cdot s}{L_{\text {Ring }}}, \beta(s)$ the horizontal beta function.

Expression (3) contains a summation over an infinite number of oscillating harmonics. However, at slow extraction, the machine operation point is brought very close to the resonance, so there is a harmonic number $N$ such that the detune value

$$
\delta=\left(\nu-\frac{N}{3}\right)
$$

is very small. In this case, harmonic $N$ is the only one that is persistent over many turns of the beam around the ring, so we ignore the others [13]:

$$
H_{1}(\phi, J)=\delta \cdot J+(2 J){ }^{1.5} G_{3, N} \cos \left(3 \Phi+\xi_{3, N}\right) .
$$

Note that we used here a new canonical variable $\Phi=\phi-\frac{N}{3} \theta$, which is a slow function of $\theta$. Equation (6) defines the shape of the extraction phase space. The design operation point in the DR is $\nu_{x}=9.65$; therefore, the resonance harmonic is $N=29$. With the design choice of magnet locations, as obtained in the simulations, the extraction phase space is shown in Fig. 3.

There are two clearly different regions in this plot. All particles inside the central triangle are stable and move around the center on closed trajectories. The maximum amplitude allowed in this triangle is

$$
2 J_{*}=\left(\frac{\delta}{3 G_{3, N}}\right)^{2} .
$$

The region outside this triangle is unstable. Particles just outside of the stable region are drifting turn by turn away from the center along the three separatrix branches, until they get to the extraction septum location, shown between the vertical dashed lines (highlighted with green), where they are extracted. On each consecutive turn, an unstable particle appears on a different separatrix branch, and it comes back to the same one again after three turns. We will refer to the branch that extends to the extraction area as the extraction branch. The first (right) vertical dashed line is the location of the septum plane that separates the region of circulating beam from the region of high electrostatic field. The increase of the amplitude from $X_{\text {sep }}$ on the extraction branch of the separatrix during three turns is called the "step size." The second (left) vertical dashed line is an imaginary boundary that shows the limitation of the machine aperture. The step size is determined by the strength of the sextupole field $G_{3, N}$. The beam losses occur due to scattering on the septum plane. Therefore, for the

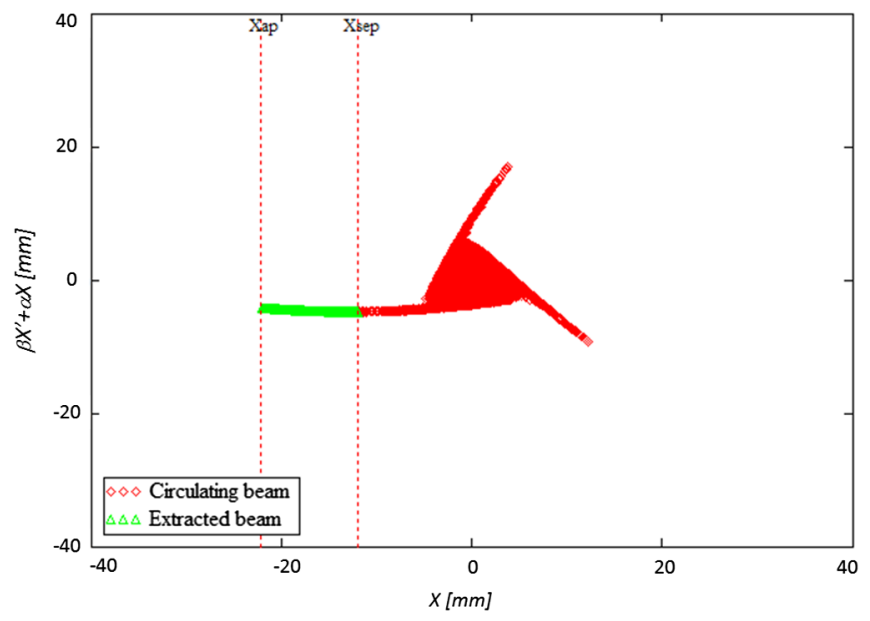

FIG. 3. The phase space at the septum location obtained with the design sextupole configuration (six magnets). The extracted part (on the left of the Xsep marker) is highlighted with green. 
best efficiency, the step size must be maximized. On the other hand, the step size is usually limited by the machine aperture $X_{\mathrm{ap}}$. To minimize the particle phase density in the septum plane, the separatrix orientation is chosen such that the extraction branch crossing $X_{\text {sep }}$ is parallel to the $X$ axis in the normalized coordinates. This fully constrains both the sextupole strength $G_{3, N}$ and its phase $\xi_{3, N}$ in Eq. (6), as well as the lower limit of the extraction losses. We will now consider extending those constraints and, therefore, pushing this limit down by the addition of dedicated multipole fields to modify the shape of the separatrix extraction branch.

\section{EFFECTS OF ADDITION OF A HIGHER MULTIPOLE}

Let us consider the basic sextupole configuration with the addition of one octupole element at an arbitrary location. The extension to the Hamiltonian form in Eq. (6) now is

$$
\begin{aligned}
H_{2}(\phi, J)= & \delta \cdot J+(2 J)^{1.5} G_{3, N} \cos \left(3 \Phi+\xi_{3, N}\right) \\
& +(2 J)^{2}\left\{\sum_{n} G_{4, n} \cos \left(4 \phi-n \theta+\xi_{4, n}\right)+\mathrm{LH}\right\} .
\end{aligned}
$$

Without going into too much detail, we just note that it is very similar to Eq. (3) and parameters $G_{4, n}$ and $\xi_{4, n}$ have similar meaning. There is also a lower harmonic part (LH) that contains second-harmonic and zero-harmonic terms. The zero-harmonic term leads to the amplitude-dependent tune shift, and the second harmonic also contains a summation over an infinite sequence $n$. It is important that the octupole term grows faster than the sextupole one with $J$, and it is important to follow up with phase shifts $\xi_{m, n}$, as those hold information about the magnet locations.

However, Eq. (8) is not taking us any further, as will be clear from the following, because all harmonics $n$ in this equation are equally important. Instead, we will approach this from a different direction, and tracking simulations give us a good hint. Let us consider several cases with a single octupole magnet placed at arbitrary locations. Figure 4 shows modified separatrices for each of those octupole locations as compared with the initial separatix shape with no octupole (red). For each octupole location, its field mostly affects the shape of just one of the separatrix branches, not all of them at once. For our purposes, we are looking for the one that acts on the extraction branch. We found that the bending of the extraction branch occurs when the octupole is placed at locations $\mu_{k}$, defined as phase advances in units of $2 \pi$ :

$$
\mu_{k}=0.10+0.5 \cdot k, \quad k=0, \pm 1, \pm 2, \ldots
$$

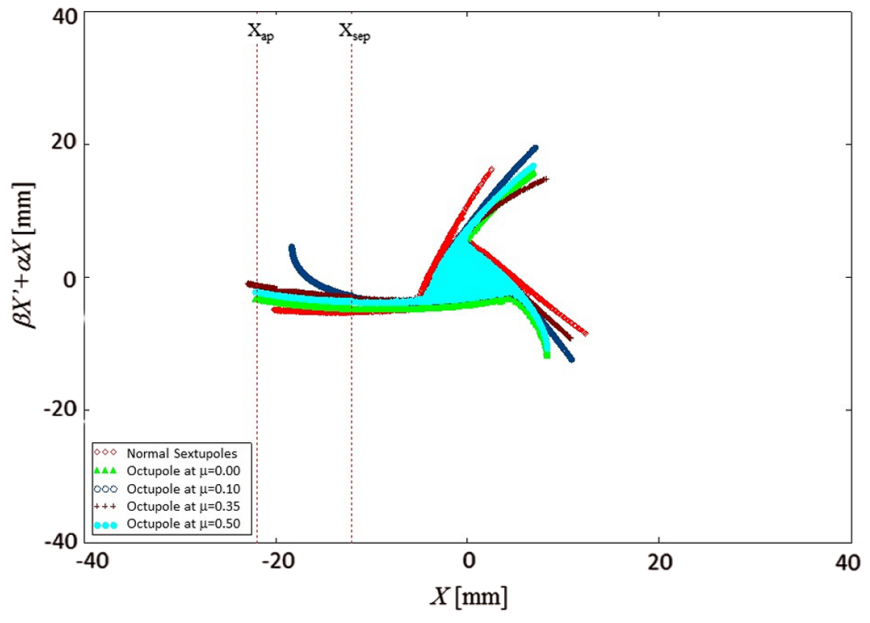

FIG. 4. Effect of an octupole magnet, placed at different locations in the ring, on the regular slow extraction phase space.

To better understand the nature of this effect, let us track back the phase space to the location of an octupole magnet. Figure 5 shows the phase space for $\mu=0.1$ at the septum (green) and at the location of the octupole (blue). One can see that, at the octupole, the extraction branch is aligned along the $X$ axis, which makes its kick the most effective. This simply reflects the fact that locations described in Eq. (9) have a phase advance to the septum close to (but not exactly) a multiple of $180^{\circ}$.

As particles enter into resonance and start to follow trajectories along the separatrix arms, the motion is dominated by the action of the sextupoles. The separatrix and separatrix arms are not significantly distorted by the octupole moments, as can be seen in Fig. 5, left. Only as the particles reach large amplitude will the octupoles have any significant action. In fact, we have found that the octupole field can be adjusted such that most of its action comes in the last turn before extraction-this can be seen from the fact that the nonextracting branches do not show significant bending. Indeed, if we disable the octupole kick on the very last turn (Fig. 5, right), all the bending action disappears. This justifies the treatment of this action as a single turn octupole kick according to Eq. (1). By treating the action as a single turn mapping, a trivial and intuitive method can be used to apply a third-order polynomial bending of the straight line using known coefficients.

Folding the extraction branch removes the constraint on the sextupole strength, and we are now able to further increase it. Without the octupole field, such a strength increase would otherwise lead to a strong overlap with the aperture, as seen in Fig. 5, right. As a result, the distribution of beam density at the septum is changing as shown in Fig. 6. Reduction of the beam density at $x=X_{\text {sep }}$ (the right boundary of the plot) leads to a reduction of the fraction of scattered beam in the septum plane by $45 \%$. The distributions are normalized such that they show the fraction of the beam passing through $50 \mu \mathrm{m}$ bins. 

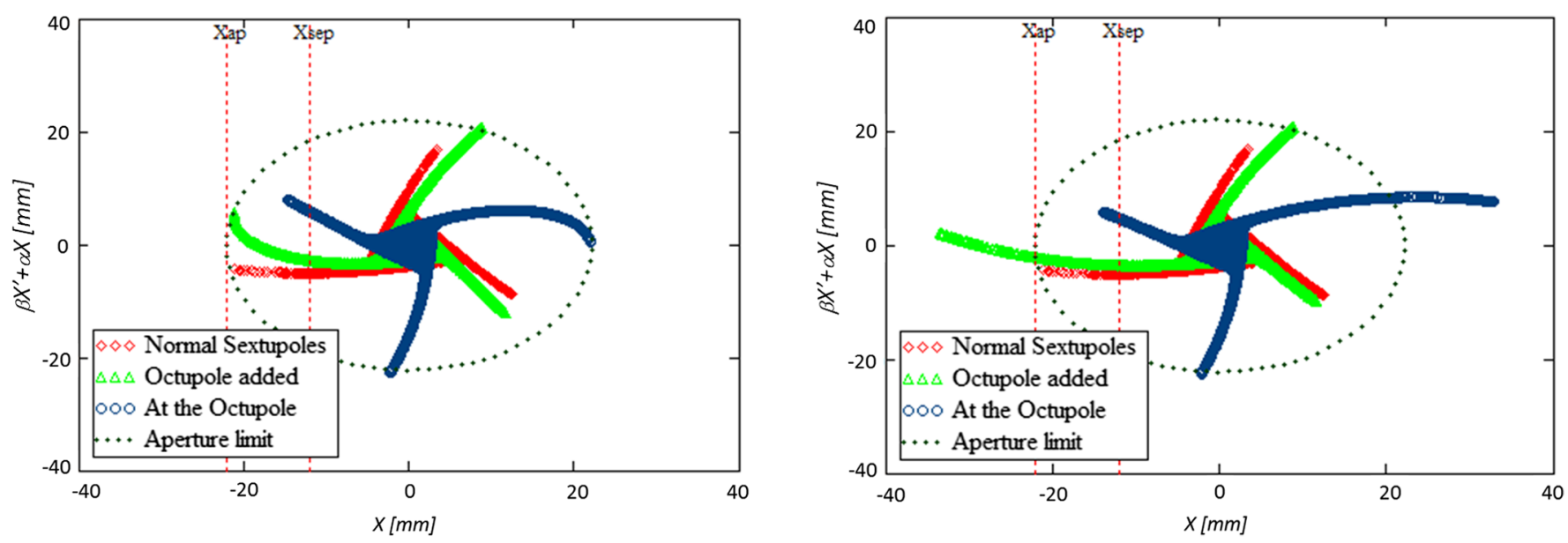

FIG. 5. Left: Phase space of the slow extraction with the addition of an octupole magnet at the optimal location $\mu=0.1$ (green). The red shape shows the normal separatrix with no octupoles, and phase space at the location of this octupole is shown in blue. The dashed ellipse shows the full machine aperture limitation. Right: Octupole kick is disabled only for the very last turn.

The integrated octupole gradient here was $1.8 \times$ $10^{4} \mathrm{~T} \mathrm{~m}^{-2}$. Typically, existing octupole magnets are substantially weaker than that, but it would not be difficult to build such a magnet. For the $1 \mathrm{~m}$ length and $10 \mathrm{~cm}$ pole-topole distance, the maximum $B$-field strength at the poles would be $0.4 \mathrm{~T}$, which is well below the normal steel saturation limit.

The efficiency improvement would not come for free, however. Bending the phase space of the extracted beam results in an emittance increase. Figure 7 shows the inscribing emittance areas for the extracted beam in the normal case (red) and the octupole-assisted case (blue). The full beam normalized emittance increases by $100 \%$, which could be an issue for some applications. Caution should be also taken to not bend the extraction separatrix

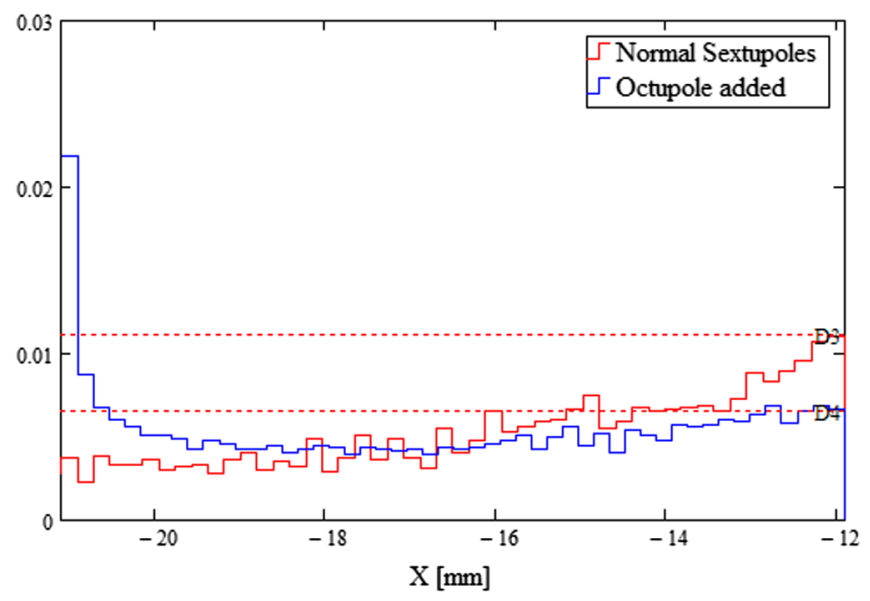

FIG. 6. Extracted beam density in $X$. The red line shows the normal extraction, and the blue line shows extraction with an additional octupole. Dashed lines and markers D3 and D4 are pointing to the beam density at the septum (right edge). The density $D 4$ is reduced by $45 \%$ compared to $D 3$. branch too much. It should not be allowed to bend back and cross the septum line again. All branches must stay within the full aperture limit, shown as a dashed line in Fig. 5 to avoid distributed losses around the ring.

Similar modifications to the extraction separatrix shape may be achieved with higher multipoles as well, using the same technique. Figure 8 shows similar performance demonstrated with a duodecapole magnet. Higher multipoles may provide a better control to the separatrix shape due to their faster field rise, but for the same reason they may require a much tighter aperture due to high magnetic field limitations on the pole tips. For example, the duodecapole magnet, in our case, would limit the diameter of beam pipe at $76 \mathrm{~mm}$ to keep the $B$ field on the pole face below $1 \mathrm{~T}$.

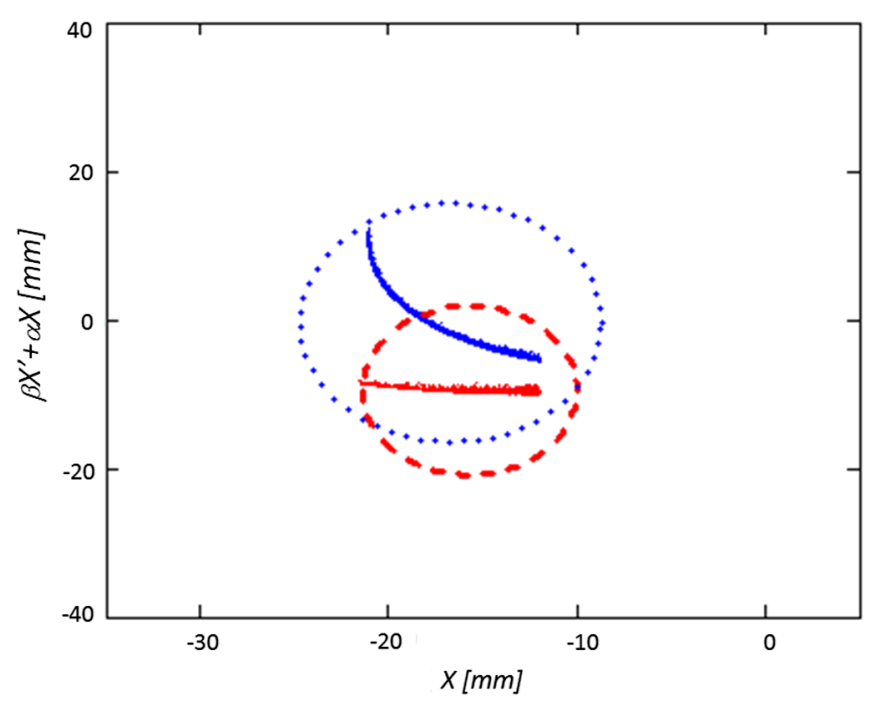

FIG. 7. Extracted beam phase space and the inclusive ellipses that show the resulting emittances. 


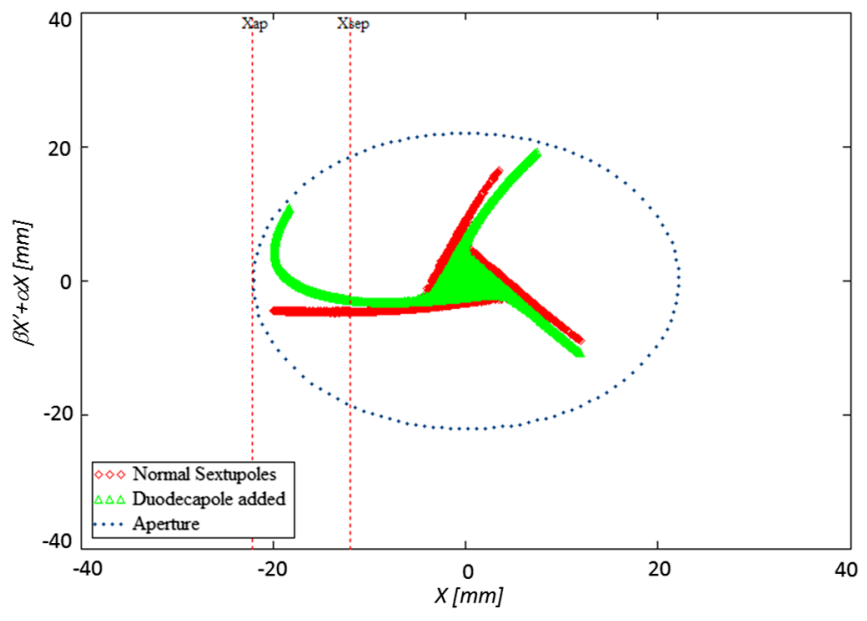

FIG. 8. Phase space modified with an addition of a duodecapole magnet.

\section{EXPLORING EFFECTS OF NATURAL HIGHER-ORDER TERMS}

The shape of the Hamiltonian in Eq. (6) is independent of whether there are multiple sextupoles in the machine or just one. Nevertheless, it is known that, to produce such a regular shape of the phase space with nearly straight separatrix branches like in Fig. 3, we need a special arrangement of many sextupole magnets. If, instead, we use only a single sextupole, configured to produce the same complex harmonic strength $\hat{G}_{k, n}$, then its phase space would appear as in Fig. 9. A good example of phase space produced with a single sextupole can be also found in Ref. [5].

Indeed, Eq. (6) is only the first-order approximation of the full Hamiltonian, used to obtain an approximate solution of motion. In higher orders of the PT corrections, the Hamiltonian will contain higher-order terms.

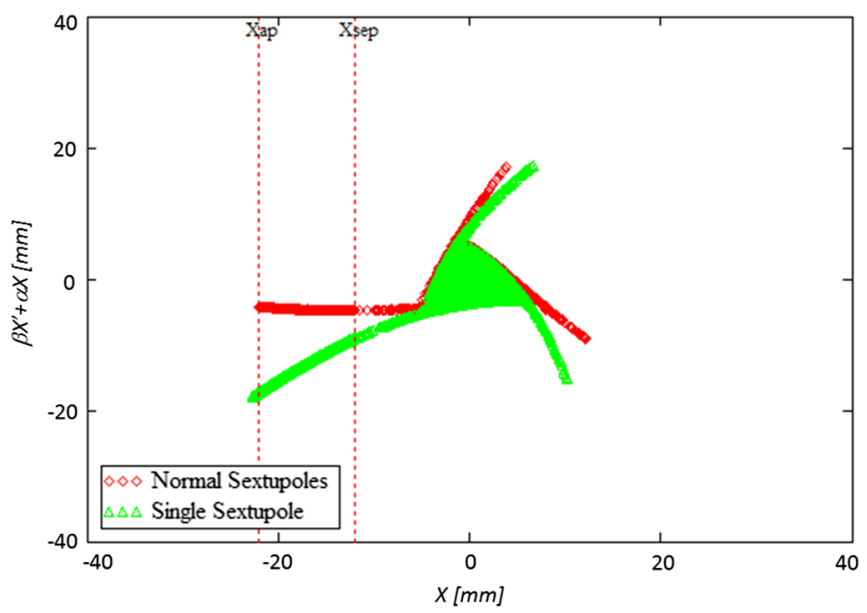

FIG. 9. Phase space for the slow extraction compared with that of an imaginary single sextupole magnet (green), producing the same resonant harmonic strength and phase.
According to Ref. [14], the second-order correction to the Hamiltonian would be

$$
\begin{aligned}
\Delta H_{2}= & \frac{3}{2}(2 J)^{2} \frac{G_{3,29}}{\delta}\left\{G_{3,29}+\sum_{n} G_{1, n-29}\right. \\
& \left.\cdot \cos \left[4 \phi-n \theta+\xi_{3,29}+\xi_{1, n-29}\right]\right\} .
\end{aligned}
$$

Here, we left only the most significant terms containing $1 / \delta$. This looks a lot like adding an octupole in Eq. (8). The first term in Eq. (10) is the zero-harmonic octupole tune shift. It cannot be changed if $G_{3,29}$ is constrained. But the next, time-dependent (in this case, $\theta$-dependent) octupole term contains $G_{1, n}$ coefficients that can be changed independently on $G_{3,29}$.

Equation (10) tells us something important: There are higher-order effects, they play a role, and these effects can be controlled by changing the configuration of the sextupoles in the machine. However, it would be difficult to use this equation to derive some practical recipe. One reason is that each of the infinite number of octupole harmonics in Eq. (10) has a different strength and phase, and quickly oscillating harmonics do not average out, because we need to consider their actions that play as fast as a single turn. There are also further higher-order effects. Starting with the second-order PT, each next order is coming with its own resonance enhancement factor $1 / \delta$, and those corrections are not vanishing. It is also important to note that, despite the amazing stability of the first-order PT model, we are studying the area of very large amplitudes, where the validity of the PT is not justified.

Instead, we studied the effects of changing the configuration of the sextupoles in the ring, using tracking simulations. There is no simple recipe in this case to optimally reconfigure sextupoles to produce the desired changes in the separatrix shapes. However, there are a few considerations that helped to simplify this search.

The space to install sextupoles in DR is available only in straight sections. Straight section SS-20-30 and part of the SS-40-50 are fully packed with injection and extraction devices and the abort line. There is a great deal of degeneracy in choosing locations within the remaining space, because the accumulated phase advance deviation from the average $60^{\circ}$ in a cell is small within one straight section. Therefore, effectively all available configurations of sextupoles near the focusing quadrupoles are equivalent to simply changing the powering scheme in the existing design geometry. In addition, we chose to preserve the condition that all magnets within one family are powered with the same supply. We are eventually coming to the limited number of combinations in which one of the three magnets in each of the two families is removed. It is worth noting that in the standard configuration the most essential $G_{1, n}$ harmonics are close to zero, and this may partially explain the smooth shape of the design phase space. 


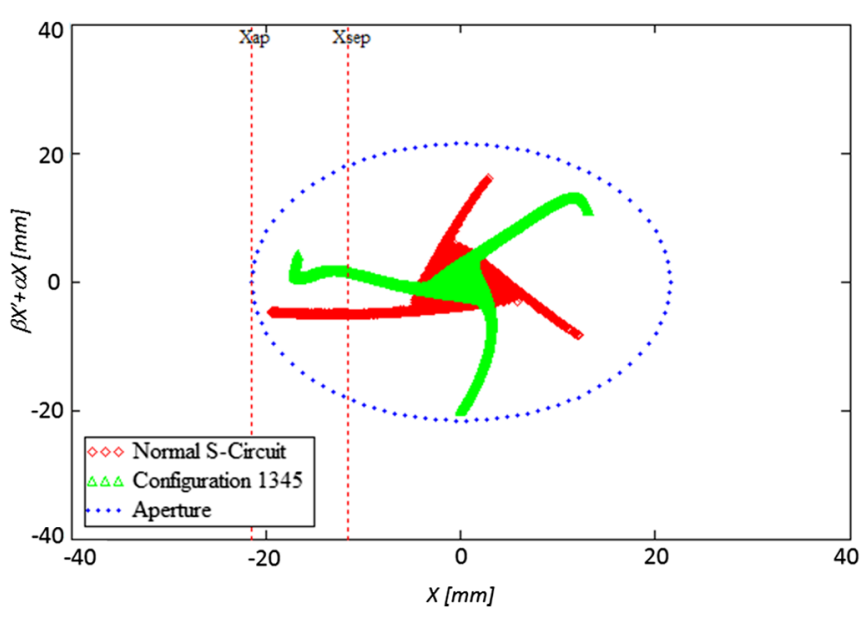

FIG. 10. Extraction phase space with the modified powering scheme in the standard sextupole configuration. Only magnets 1 , 3,4 , and 5 are powered, and the strength is increased. The dashed line shows the machine acceptance.

Removing one out of three magnets in each family gives rise to $G_{1, n}$-s and, therefore, the higher-order effects. From these combinations, we found a configuration " 1345 " of four magnets out of six ("1345" means that magnets " 2 " and " 6 ," or second in the first circuit and third in the second circuit are missing; configuration " 123456 " would mean all six magnets are present). With the adjustment of the relative strengths between the families, the extraction phase space can be brought to that in Fig. 10. The effective reduction of the beam losses in this configuration is $30 \%$, whereas the extracted beam emittance is also reduced by $45 \%$, unlike in the cases considered above. An interesting new feature of the phase space is the two kinks in the extraction branch, which suggest that there is more than one higher perturbation order involved. Again, the separatrix folding changes the constraints, and this setup involves a substantial increase of the individual sextupole strength, more than a factor of 3, which is beyond the capability of existing magnets and power supplies. Further increase of the magnet strength and curving of the extraction branch is limited by the rapid growth of the other separatrix branches and reaching the limit of the machine aperture. Note that we restricted the variability of considered sextupole configurations in this search to a limited number of practical, simple combinations. One more thing that we left out of the search is moving the sextupoles away from the focusing quadrupoles. This can be a very powerful degree of freedom, because it gives great control on the phase contribution of each magnet.

\section{BENCHMARKING}

Although the simulation approach with simple 2D tracking is quite adequate in showing the underlying nonlinear dynamics, it is necessary to benchmark those simulations with a more detailed $6 \mathrm{D}$ tracking. This has been done using the universal pyOrbit code developed jointly by Oak Ridge National Laboratory and CERN [15]. There are several important differences in performance introduced by the extension of the tracking to all six dimensions. The natural chromaticity of the DR FODO lattice is large, and we chose to set the (unnormalized) chromaticity to the design value of $Q^{\prime}=2.0$ using all of the 138 existing arc sextupoles. These sextupoles are placed with a mirror symmetry in each arc, and arcs follow the overall threefold symmetry of the DR. This effectively suppresses third-, fourth-, and fifth-order resonance strengths of the chromaticity sextupoles compared to the main slow extraction sextupole circuit, but it does not suppress the nonresonant and higher-order harmonics. The beam momentum spread is determined by the rf manipulations in the Recycler ring and is expected to have rms $=0.15 \%$. This spread was introduced in the beam sample with the Gaussian distribution, matched with the corresponding longitudinal distribution and tracked with the design Delivery Ring rf field. Momentum spread contributes to the angular spread of the extracted beam and, therefore, adds to the septum losses. This contribution is expected to be small, as we are choosing very low chromaticity values. We also chose to include the beam space charge effect, corresponding to $10^{12}$ protons in the bunch. It turned out that the most limiting factor for substantial increasing of the main driving sextupole field strength is the coupling between the horizontal and vertical motion, even though we were careful to keep the vertical machine tune away from major coupling resonances. In fact, this sort of coupling instability takes place within the last 1-3 turns; therefore, it has a broad band nature and is contributed by all existing high-order harmonics. Having a small initial vertical emittance tremendously helps to avoid this destructive coupling, so it may not be an issue with lower emittance machines. With the $2.6 \pi$-mm-mrad normalized $\mathrm{rms}$ emittance, $8 \mathrm{GeV}$ beam in the DR, this coupling puts a limitation on the maximum sextupole strength and resulting phase space shapes. With all these additions and limitations, we have still been able to observe that the DR sextupole configuration 1345 produces the desired bend in the extraction branch of the separatrix, although the module and relative strengths between the two magnet families had to be readjusted. At this time, the separatrix bending allowed a further sextupole strength increase of more than a factor of 2 .

The resulting phase space is shown in Fig. 11. The red points show the snapshot locations of the circulating beam macroparticles, and the green dots show the extracted portion of the beam at the septum for the nominal configurations 123456. Blue dots show the extracted beam phase space in the modified geometry 1345 . Note that the substantial visible angular spread in the extracted beam appears due to the relatively long time to accumulate this sample, as the dynamic orbit bump compensation was not 


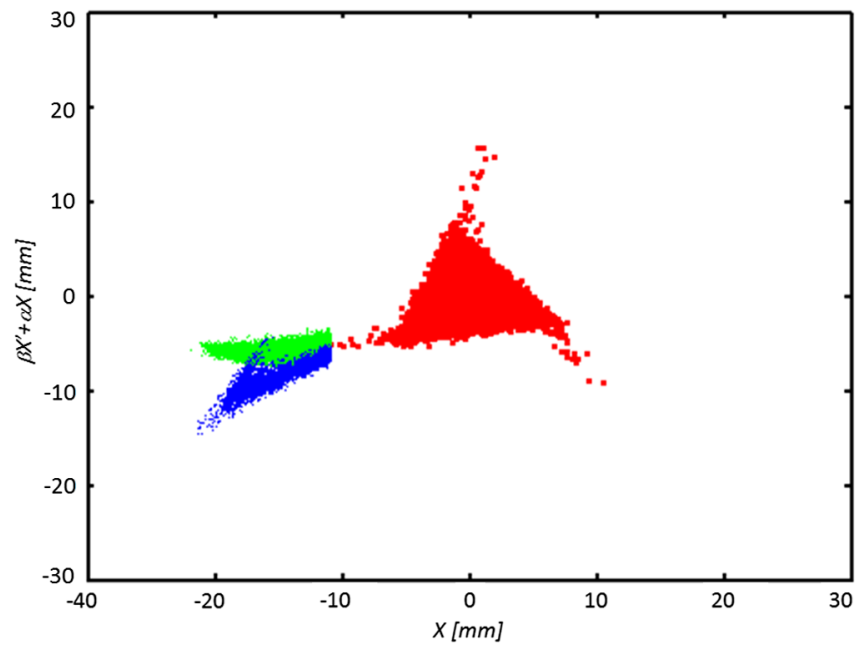

FIG. 11. Folding the extraction separatrix branch in the pyOrbit tracking simulations. Extracted beam samples are collected over many turns.

included in this tracking. Figure 12 shows the $X$ distributions for the extracted beam in the two configurations. The distributions are normalized such that they show the fraction of the beam passing through $50 \mu \mathrm{m}$ bins.

The density reduction for the $50 \mu \mathrm{m}$ wide septum plane in this case is $25 \%$, and the extracted beam full emittance, unlike in Fig. 10, is grown by $27 \%$. It is interesting to note here the phase shift of the extraction branch compared to the reference. This can be corrected by adjusting the phase advance between the effective sextupole kick and the septum. However, it was more difficult to do this adjustment within the parameter space that we chose to manipulate, because changing the relative strengths of the two sextupole families now also has the effect of changing the effective higher-order content. We did not pursue this further at this point, although this produces an effect of reduced step size projection on the $X$ plane and the

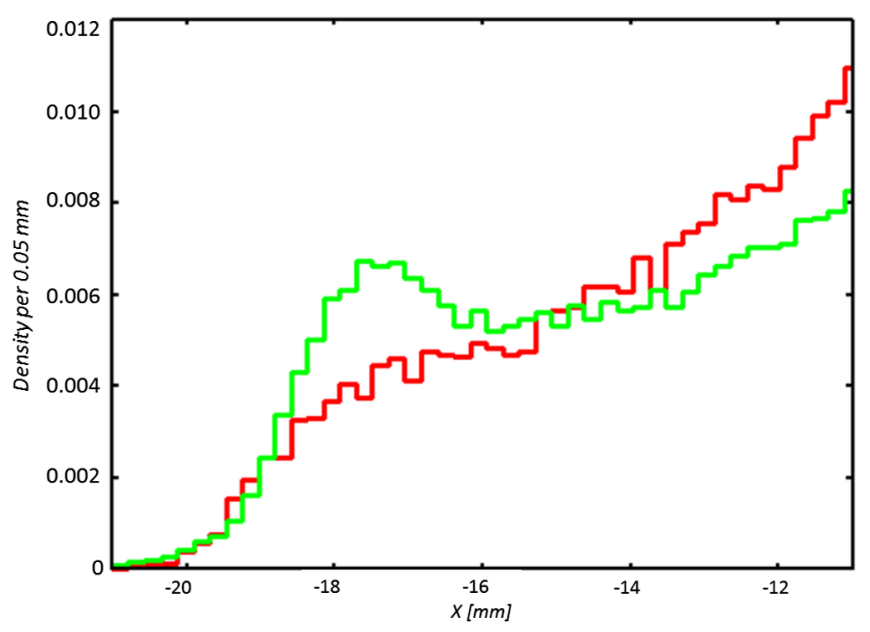

FIG. 12. $X$ histogram of the extracted beam for configurations 123456 (red) and 1345 (green).

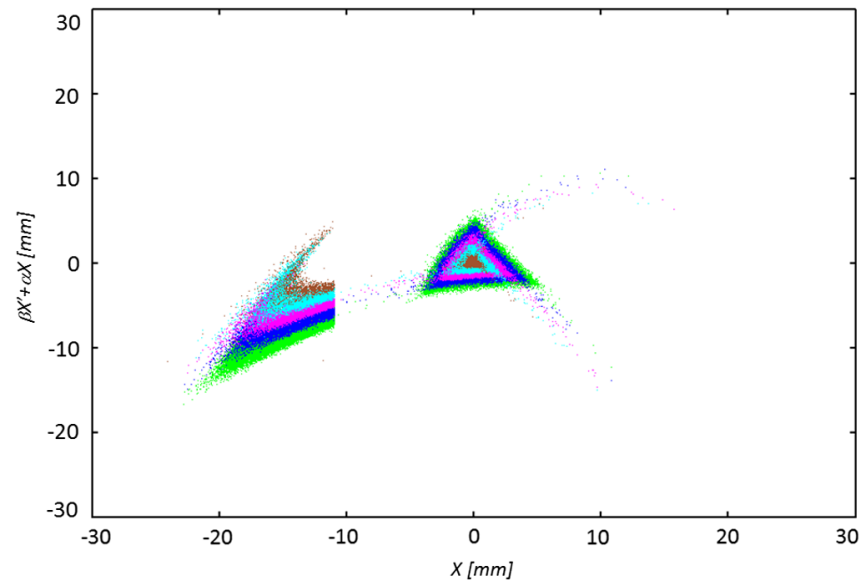

FIG. 13. Separatrix shape evolution during the entire spill. Different colors show the phase space at different moments of the spill.

same-order effect of increasing the angular spread. The effect of separatrix folding remains through the entire spill as the tune is moved to the exact resonance, although the folding pattern is changing. Figure 13 shows the evolution of the phase space through the spill. The space occupied by the extracted branch is squeezing in $X$ with time. This is seen in the density distribution evolution through the spill shown in Fig. 14. The red curve is the distribution for the standard configuration, and the others are the distributions at different moments of the spill for the configuration 1345 . The density at the septum plane changes very little with the tune. Squeezing the distributions to the right moves the extracted beam phase space footprint away from the acceptance boundary and, thus, may leave a possibility to further improve them by ramping up the sextupole strength. This was not studied here at this time.

The case of an additional octupole, considered in Sec. V, is more straightforward and intuitive than the pure

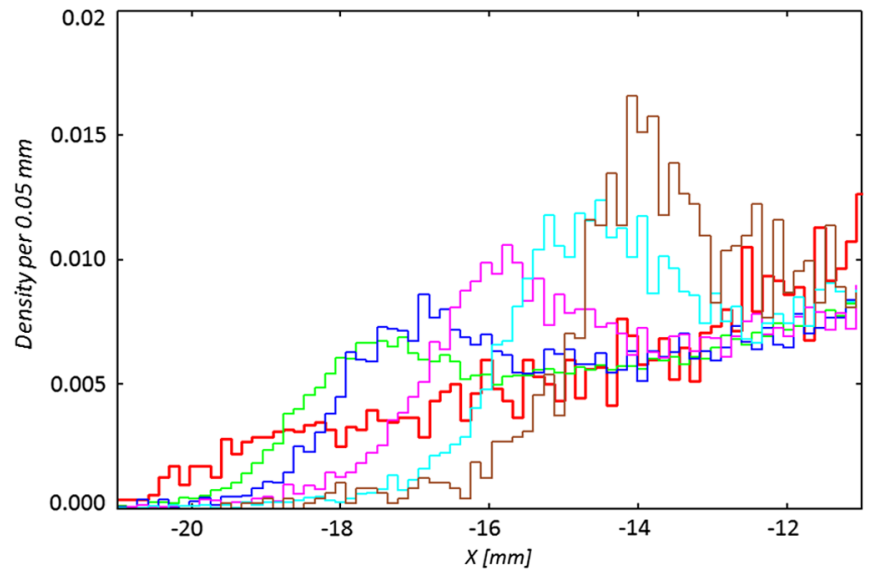

FIG. 14. Evolution of the spatial density in the extracted beam during the spill. The red curve shows the distribution for the standard design configuration. 


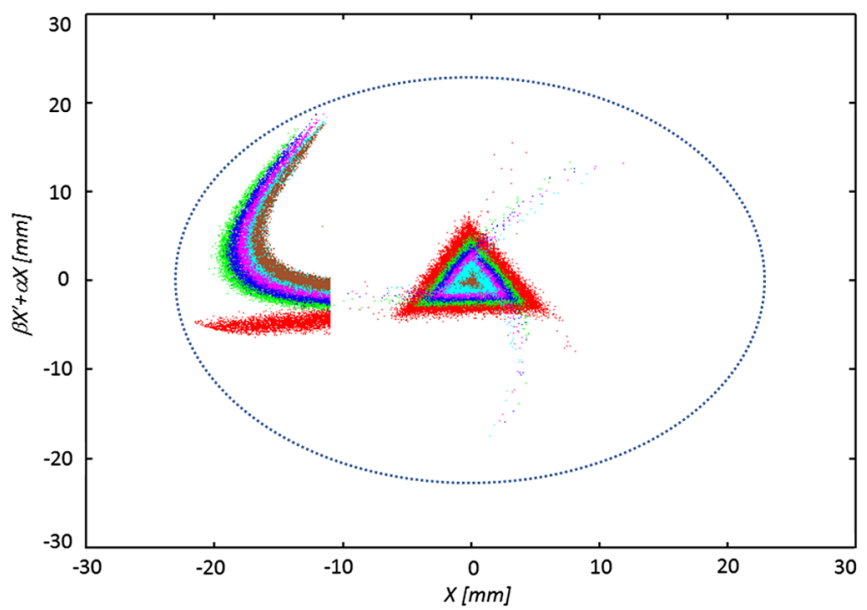

FIG. 15. Separatrix folding with the addition of an octupole magnet at different moments during the spill. The red shapes show the undisturbed phase space (no octupole). The extracted beam samples are accumulated over many turns.

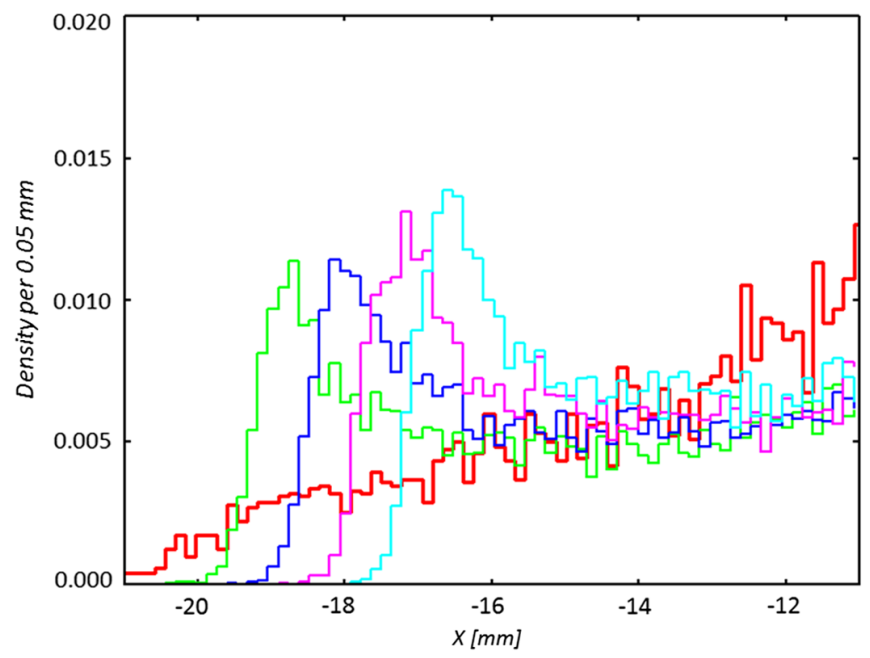

FIG. 16. Evolution of the horizontal distribution of the extracted beam with time during the spill. The red histogram shows the undisturbed distribution.

sextupole configurations. Nevertheless, we also performed a similar benchmarking for the final octupole configuration with $6 \mathrm{D}$ tracking and verified similar results. Figure 15 shows the phase space with the addition of an octupole in the straight section 40 . The extracted beam horizontal distributions across the spill are shown in Fig. 16. Density reduction at the septum, leading to the beam loss reduction of $40 \%$, stays approximately constant during the spill.

\section{CONCLUSIONS}

Using simple tracking simulations, we have demonstrated that an addition of a single higher multipole magnet can be very effective to reduce the extraction beam losses originating from scattering on the septum. A simple and effective recipe is provided for the best choice of this magnet location. A reduction of beam losses up to $40 \%$ can be achieved by using an octupole magnet. The use of the higher than $m=3$ multipoles is also possible but may be limited because of higher field strength at the magnet pole tips. We also explored the very interesting option of using an intrinsic higher-order harmonic content that is present in the sextupole configurations. We have shown that it is possible to produce a similar effect with simply changing the powering scheme in the existing lattice. In this case, 2D simulations were instrumental to demonstrate the effect, but the final geometry needed to be readjusted using full 6D simulations to take into account several other realistic operational conditions.

\section{FURTHER DISCUSSION}

There are two operational factors that significantly limit the freedom of controlling the separatrix folding: the machine acceptance and beam blowup due to the fast coupling instability. In our case, both were important, and the latter one certainly deserves a separate study. There are also other practical limitations specific to the Delivery Ring. Although we limited the sextupole field changes to a simple powering reconfiguration, the realization of the new sextupole scheme in practice would require substantial additions. The maximum individual sextupole strengths need to be increased by at least a factor of 2 , which requires adding more magnets and augmenting the power supplies. It will also be necessary to add more ramping quadrupole magnets to keep up with the stronger sextupole field. So, adding a single multipole magnet may appear to be a more practical solution, if extracted beam emittance growth is acceptable. This does not rule out the possibility of using the sextupole configurations to achieve an effective separatrix folding. We did not explore the full range of configuration changes. In this respect, it would be very interesting to try a similar study for other machines where there are fewer limitations in the existing lattice configurations. We are also exploring the options to experimentally test some of the higher-order effects in electron machines with pencil-size beams.

\section{ACKNOWLEDGMENTS}

We are very grateful to Dr. Leo Michelotti for his interest to the subject and very fruitful discussions. This work has been performed under Contracts No. DE-AC0207CH11359, No. DE-SC0012704 and Grant Award No. LAB 18-1802 with the auspices of the U.S. Department of Energy, also funded by the Japan High Energy Accelerator Research Organization and the Japan Society for the Promotion of Science.

[1] K. A. Brown, V. Schoefer, and M. Tomizawa, Improving extraction efficiency of the third integer resonant extraction using higher order multipoles, BNL, Upton, NY, Technical Report No. C-A/AP/584, 2017. 
[2] N. N. Alexeev, S. L. Bereznitsky, and A. E. Bolshakov, Perturbation of relevant resonance for slow extraction efficiency increase, in Proceedings of the Particle Accelerator Conference, Vancouver, BC, Canada, 1997 (IEEE, New York, 1997), pp. 261-263.

[3] L. S. Stoel et al., Phase space folding studies for beam loss reduction during resonant slow extraction at the CERN SPS, in Proceedings of IPAC-2017, Copenhagen, 2017 (JACoW, Geneva, Switzerland, 2017), pp. 615-618, MOPIK046, http://accelconf.web.cern.ch/AccelConf/ ipac2017/doi/JACoW-IPAC2017-MOPIK046.html.

[4] L.S. Stoel et al., Investigating beam loss reduction with octupoles during slow extraction in the CERN SPS, in Proceedings of IPAC2018, Vancouver, BC, Canada, 2018 (JACoW, Geneva, Switzerland, 2018), pp. 822-825, TUPAF051, http://accelconf.web.cern.ch/AccelConf/ ipac2018/doi/JACoW-IPAC2018-TUPAF051.html.

[5] K. Brunner et al., Use of a massless septum to increase slow-extraction efficiency, in Proceedings of IPAC2018, Vancouver, BC, Canada, 2018 (JACoW, Geneva, Switzerland, 2018), pp. 862-865, TUPAF061, http://accelconf .web.cern.ch/AccelConf/ipac2018/doi/JACoW-IPAC2018TUPAF061.html.

[6] International Workshop on Slow Extraction, https://indico .cern.ch/event/639766/, CERN, 2017.

[7] L.S. Stoel, Low-loss resonant extraction from hadron synchrotrons in the TeV energy scale, Ph.D. thesis, Vienna University of Technology, 2019.
[8] M. Tomizawa, Y. Arakaki, T. Kimura, R. Muto, S. Murasugi, K. Okamura, H. Sato, Y. Shirakabe, and E. Yanaoka, Slow extraction from J-PARC Main Ring using a dynamic bump, Nucl. Instrum. Methods Phys. Res., Sect. A 902, 51 (2018).

[9] M. Tomizawa et al., Status and beam power ramp-up plans of the slow extraction operation at J-PARC Main Ring, in Proceedings of the HB-2018, Daejeon, Korea (JACoW, Geneva, Switzerland, 2018), pp. 347-351, THA1WD03, http://accelconf.web.cern.ch/AccelConf/hb2018/doi/JACo W-HB2018-THA1WD03.html.

[10] L. Bartoszek et al., Mu2e technical design report, arXiv: 1501.05241.

[11] V. Nagaslaev et al., Measurement and optimization of the lattice functions in the debuncher ring at Fermilab, Report No. FERMILAB-CONF-06-200-AD, 2006, http://accelconf.web.cern.ch/AccelConf/e06/PAPERS/ WEPCH057.PDF.

[12] L. Michelotti and J. Johnstone, Preliminaries toward studying resonant extraction from the debuncher, Report No. FERMILAB-FN-0842-APC-CD, 2009.

[13] S. Y. Lee, Accelerator Physics (World Scientific, Singapore, 1999).

[14] S. Ohnuma, Quarter integer resonance by sextupoles, Report No. Fermilab TM-448, 1973.

[15] A. Shishlo, S. Cousineau, J. Holmes, and T. Gorlov, The particle accelerator simulation code PyORBIT, Proc. Comput. Sci. 51, 1272 (2015). 